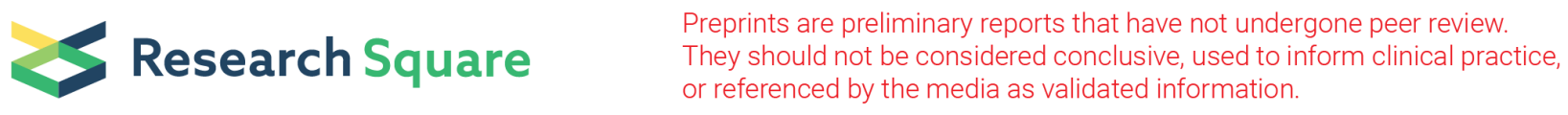

\title{
Bacterial Profile and Drug Susceptibility among Adult Patients with Community Acquired Lower Respiratory Tract Infection at Tertiary Hospital, Southern Ethiopia
}

\author{
Alemitu Beyene Gebre \\ Hawassa University College of Medicine and Health Sciences \\ Tsegaye Alemayehu Begashaw \\ Hawassa University College of Medicine and Health Sciences \\ Moges Desta Ormago ( $\sim$ moges22@yahoo.com) \\ Hawassa University College of Medicine and Health Sciences
}

\section{Research article}

Keywords: Lower Respiratory Tract Infection, culture-positive, Antimicrobial susceptibility test

Posted Date: January 8th, 2021

DOI: https://doi.org/10.21203/rs.3.rs-141244/v1

License: (c) (1) This work is licensed under a Creative Commons Attribution 4.0 International License. Read Full License 


\section{Abstract}

Background: Lower respiratory tract infection is a global problem accounting over 50 million deaths annually. Here, we determined the bacterial profile and antimicrobial susceptibility pattern of lower respiratory tract infections among adult patients attending at Tertiary Hospital, Southern Ethiopia.

Methods: A cross sectional study was conducted among adult patients with lower respiratory infection at the medical outpatient department of the Hospital. A sputum sample was collected and processed for bacterial culture and antimicrobial susceptibility test. Semi structured questionnaires were used to collect data. SPSS version 22 software was used for statistical analysis and a $p$ value of $<0.05$ was considered as statistically significant.

Results: Out of 406 sputum samples of participants 136(33.5\%) were culture positive for 142 bacterial isolates. Klebsiella pneumoniae 36(25.4\%) was the predominant isolate followed by Pseudomonas species 25(17.6\%). Gram-negative bacteria were sensitive to cefepime (86.0\%) and ciprofloxacin (77.8\%) antibiotics while gram-positive (76.5\%) to clindamycin.

Conclusion: Community acquired lower respiratory tract Infection was highly prevalent in the study area and the isolates showed resistant to common antibiotics such as ampicillin, augmentin, ceftazidime and tetracycline. Therefore, culture and susceptibility test is vital for appropriate management of lower respiratory tract infection in the study area

\section{Background}

Lower respiratory tract infections (LRTIS) are one of the main respiratory diseases that challenging the world and they remain the deadliest communicable disease and the 3rd leading cause of death around the world, after ischaemic heart and cerebrovascular diseases [1]. In Sub-Saharan Africa, LRTIs rank third after HIV/AIDS and malaria in terms of causes of mortality [2].

The etiological agents of LRTI infections vary between populations and countries, depending on the difference in geography, climate, and socioeconomic conditions, associated factors of LRTI as well as their antibiotics susceptibility [3].

The initial antimicrobial management for community acquired pneumonia (CAP) is usually empirical and selecting an appropriate regimen requires knowledge of the spectrum of organisms implicated in CAP locally [4]. Therefore, studies are critical to identify the microorganisms causing LRTI in the local context and to determine their susceptibility to various antimicrobials. Initial empirical broad-spectrum therapy can then be narrowed based on the culture results [5]. This study aimed to determine the bacterial pathogens with their antimicrobial susceptibility pattern and associated factors in adult patients with LRTI.

\section{Method}

\section{Study design, period and population}

Cross-sectional study was conducted in Hawassa University Comprehensive Specialized Hospital (HUCSH), Southern Ethiopia from July to October 2019. Individual patients with age of $\geq 18$ years old having symptoms of LRTI, in particular, productive cough, fever, chest pain and acid-fast bacilli (AFB) smearnegative were included in the study. Patients who were on antibiotic treatment in the last 14 days, patients who were experienced tuberculosis in the last two years, those who were severely ill and unable to give sputum sample and those who were failed the Bartlett's criteria were excluded.

\section{Sample size determination and sampling technique}

The sample size was determined using single proportion formula,

$n=\left(z_{\alpha / 2}\right)^{2}(p(1-p)) / d^{2}$

considering the $95 \%$ confidence level $\mathrm{Cl}(\mathrm{z}=1.96)$ taking the prevalence $(\mathrm{p})$ as $40 \%$ from previous study conducted in Arba Minch [6] and margin of error (5\%), $(d=0.05)$ the formula used to calculate the sample size $(n)$ was as follow.

$$
\mathrm{n}=\frac{(1.96)^{2}(0.4(1-0.4))}{0.05^{2}}=369
$$

With contingency of $10 \%=369+37=406$

Systematic random sampling technique was applied to select 406 respondents for interview. The interval (K) was obtained by dividing the total number of patients attending the medical OPD for LRTI during the study period by sample size.

According to information found from the data base of HUCSH the average daily patient flow due to LRTI at OPD is 11 . The study period took three months, which contain 66 days and 11 times 66 gives 726 . Therefore, the total patient flow during the study period is 726 .

$K=726 / 406=1.8 \approx 2$ 
The first individual is selected by lottery method and the others at regular interval.

\section{Data collection and laboratory processing}

All relevant socio-demographic and clinical data were collected by trained nurses through face-to-face interviews with the patient using a semi structured questionnaire from 406 respondents whose sputum were AFB smear-negative and fulfill Bartlett's criteria on Gram's stain. Spot- spot sputum samples were collected with one-hour interval using dry, sterile, leak proof, translucent, and screw-capped plastic containers with a capacity of $30 \mathrm{ml}$ and brought to the Microbiology Laboratory of HUCSH for laboratory processing.

\section{Sputum Microscopy for AFB}

All Sputum samples were examined by the Light emitting diode fluorescence microscope (LED FM) for AFB detection [7]. The AFB appeared as bright yellow against dark back ground materials. Sputum smear positive cases were excluded from the study whereas sputum smear negative cases were evaluated further based on Bartlett's grading system and macroscopically [8].

\section{Gram's stain}

Their sputum samples were checked macroscopically for color, volume, viscosity, odor, and any positive score (sum of + and - values assigned) on Gram's stain was considered as acceptable result to culture

Sputum sample based on Bartlett's criteria [8]

\section{Cultivation and identification of isolates}

The purulent part of accepted sputum sample was inoculated to blood agar (Oxoid, Hampshire, UK), MacConkey agar (Oxoid, Hampshire, UK) and chocolate agar (Oxoid, Hampshire, UK) with the sterile wire loop. In order to get a single pure colony, the samples were streaked into four quadrants of the plate with flaming the loop in between each spread. The inoculated MacConkey agar plates were incubated aerobically at $37^{\circ} \mathrm{C}$ for 24 hours whereas blood and chocolate agar were inoculated using $5-10 \% \mathrm{CO}_{2}$ generating candle jar at $37^{\circ} \mathrm{C}$ for 24 hours. After 24 hours incubation, the plate was examined for growth. Pure colonies were sub-cultured to nutrient broth (Oxoid, Hampshire, UK). Finally, bacterial species were identified by using the standard microbiological technique [9].

Pure isolates of bacterial pathogens were preliminarily characterized by colony morphology, gram stain and hemolytic reactions on blood agar plates. Identification of bacteria down to species level was done by biochemical tests such as catalase, coagulase, optochin $(30 \mu \mathrm{g})$ test, bacitracin $(30 \mu \mathrm{g})$ test and bile esculin agar for Gram-positive identification and oxidase, indole production, urease, citrate utilization, lysine decarboxylation, carbohydrate fermentation, gas production, $\mathrm{H}_{2} \mathrm{~S}$ production and motility for Gram-negative bacteria [10].

\section{Antimicrobial susceptibility testing}

Antimicrobial susceptibility test (AST) was performed for identified bacterial isolates by disc diffusion technique as recommended by Clinical and Laboratory Standard Institute on Mueller-Hinton agar (Oxoid, Hampshire, UK) and 5\% sheep blood supplemented Mueller-Hinton agar for fastidious bacterial isolates.

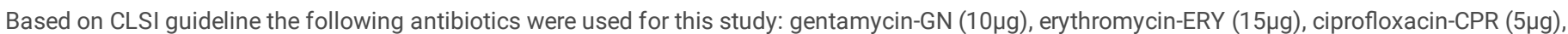
tetracycline-TAT $(30 \mu \mathrm{g})$, ampicillin-AMP $(10 \mu \mathrm{g})$, augmentin-AUG $(10 \mu \mathrm{g})$, ceftriaxone-CTR $(30 \mu \mathrm{g})$, chloramphenicol-CAF $(30 \mu \mathrm{g})$, ceftazidime-CAZ $(10 \mu \mathrm{g})$, cotrimoxazole-COT $(1.25 / 23.75 \mu \mathrm{g})$, cefepime-CEP $(30 \mu \mathrm{g})$, cefoxitin-CXT $(30 \mu \mathrm{g})$, penicillin-PEP $(10 \mu \mathrm{g})$, clindamycin-CLD $(2 \mu \mathrm{g})$ and. Finally, the result was reported as sensitive $(S)$, intermediate $(I)$ or resistance $(R)$ by measuring the diameter of zone of inhibition or hemolysis[11].

\section{Quality control}

Prior to actual data collection, the quality of data was assured by pre-testing questionnaires on 20 participants at HUCSH for assessing its clarity and to take amendments and orientation was given to data collectors. The prepared culture media were checked for sterility by incubating the five percent of prepared media for 24 hours and observed for the presence of any colony growth. In addition, the abilities of the prepared media supporting the growth of organisms were checked by inoculating control strains. Standard reference strains of S. pneumoniae (ATCC-49619), H. influenzae (ATCC-49766) E. coli (ATCC-25922), S. aureus (ATCC-25923), P. aeruginosa (ATCC-27853) and p. mirabilis (ATCC-12453) were used during culture and antimicrobial susceptibility testing

\section{Data processing and analysis}

All filled questionnaires for this study was checked visually, coded and entered into excel and then exported to SPSS version22 software (SPSS Inc., Chicago, IL, USA) for analysis. Bivariate logistic regression was used to determine predictors of culture confirmed LRTI. For those variables, which $p$-value $<0.25$ in the bivariate, the analysis was further entered into the multivariable logistic regression model[12]. Associations between dependent and independent variables were assessed and its strength was described using odds ratios at $95 \%$ confidence intervals. A statistically significant association considered as $p$-value $<$ 0.05 .

\section{Results}

\section{Sociodemographic characteristics}


A total of 406 LRTI suspected adult patients were enrolled in this study. Of them, 246(60.6\%) were male. The mean age of patients was $36.75 \pm 14.84$. More than half of the participants live in urban 225(55.4\%) [Table1].

\section{Clinical characteristics}

Among 406 study participants, twenty six (6.4\%) of participants have previous tuberculosis disease exposures before two years and $34(8.4 \%)$ of participants have heart disease and $26(6.4 \%)$ were HIV positive [Table2].

Table 1 Sociodemographic characteristics of adult patients with lower respiratory tract infection at HUCSH, 2019

\begin{tabular}{|c|c|c|c|}
\hline \multicolumn{2}{|l|}{ Variables } & \multirow{2}{*}{$\begin{array}{l}\text { Frequency } \\
246\end{array}$} & \multirow{2}{*}{$\begin{array}{l}\text { Percent (\%) } \\
60.6\end{array}$} \\
\hline Sex & Male & & \\
\hline & Female & 160 & 39.4 \\
\hline \multirow[t]{4}{*}{ Age(year) } & $18-35$ & 235 & 57.9 \\
\hline & $36-49$ & 93 & 22.9 \\
\hline & $50-64$ & 51 & 12.6 \\
\hline & $>65$ & 27 & 6.7 \\
\hline \multirow[t]{2}{*}{ Residence } & Rural & 181 & 44.6 \\
\hline & Urban & 225 & 55.4 \\
\hline \multirow[t]{4}{*}{ Education status } & No formal education & 142 & 35.0 \\
\hline & $1-8$ grade & 141 & 34.7 \\
\hline & $9-12$ grade & 70 & 17.2 \\
\hline & Diploma \& above & 53 & 13.1 \\
\hline \multirow[t]{2}{*}{ Marital status } & Single & 104 & 25.6 \\
\hline & Married & 302 & 74.4 \\
\hline \multirow[t]{6}{*}{ Occupation } & Farmer & 89 & 21.9 \\
\hline & Government & 43 & 10.6 \\
\hline & Private & 93 & 22.9 \\
\hline & Housewife & 112 & 27.6 \\
\hline & Student & 52 & 12.8 \\
\hline & Jobless & 17 & 4.2 \\
\hline \multirow{6}{*}{$\begin{array}{l}\text { Monthly income } \\
\text { (Ethiopian birr) }\end{array}$} & $<1000$ & 126 & 31.0 \\
\hline & $1000-2000$ & 79 & 19.5 \\
\hline & $2001-3000$ & 118 & 29.1 \\
\hline & $3001-5000$ & 52 & 12.8 \\
\hline & $>5000$ & 19 & 4.7 \\
\hline & No monthly income & 12 & 3.0 \\
\hline
\end{tabular}

Table 2 Clinical characteristics of adult patients with lower respiratory tract infection at HUCSH, 2019 


\begin{tabular}{|llll|}
\hline Variables & & Frequency & Percent (\%) \\
\hline Cigarette smoking & Smoker & 33 & 8.1 \\
& No smoker & 373 & 91.9 \\
\hline Alcohol drinking & Yes & 55 & 13.5 \\
& No & 351 & 86.5 \\
\hline Crowded living condition & Yes & 52 & 12.8 \\
& No & 354 & 87.2 \\
History of the previous TB & Yes & 26 & 6.4 \\
& No & 380 & 93.6 \\
Heart disease & Yes & 34 & 8.4 \\
& No & 372 & 91.6 \\
\hline Previous HIV screening & Positive & 26 & 6.4 \\
& Negative & 167 & 42.1 \\
& Unscreened & 213 & 52.5 \\
\hline
\end{tabular}

\section{Bacterial profile of LRTIs}

The overall culture-positive sputum sample from a patient with LRTI in this study were 136/406(33.5\%), 95\% Cl (28.8\%-40\%) with a total bacterial isolate of $142 / 406(35.0 \%)$. Single bacterial pathogens were isolated from 130(32.0\%) patients, while mixed infections were isolated from 6(1.4\%) patients. Among mixed infection isolate, E. coli and S. aureus were isolated from 1(0.2\%) patient, E. coli and Pseudomonas spp from 1(0.2\%) patient and K. pneumoniae and E. coli from 4(1.0\%) patients. The gram-negative bacteria 108(76.0\%) predominated over gram-positive bacteria. In this study, K. pneumoniae $36(25.4 \%)$ was the frequently isolated bacteria followed by Pseudomonas spp 25(17.6\%), E. coli 22(15.5\%) and S. aureus 21(14.8\%) [Table3].

Table 3 Frequency of bacterial isolates identified from sputum specimen of an adult patient with community acquired LRTI at HUCSH 2019

\begin{tabular}{|lll|}
\hline Isolates & Frequency & Percent (\%) \\
\hline K. pneumoniae & 36 & 25.4 \\
\hline Pseudomonas spp & 25 & 17.6 \\
\hline E. coli & 22 & 15.5 \\
\hline S. aureus & 21 & 14.8 \\
\hline Acinetobacter spp & 19 & 13.4 \\
\hline S.pneumoniae & 9 & 6.3 \\
\hline Enterobacter spp & 4 & 2.8 \\
\hline Others* & 6 & 4.2 \\
\hline
\end{tabular}

\section{* Citrobacter spp, S.pyogene and Enterococcus spp}

The bacterial pathogens were more predominant in male $87(64.0 \%)$ than female as the study result shows. The age group $18-35$ years was more susceptible to bacterial pathogens of LRTI $64(47.1 \%)$ than other age groups. Of 302 married study participants enrolled in this study, 112(82.4\%) were positive for sputum culture. Among 43(10.6\%) government workers participated in study 11(8.1\%) were positive for bacterial pathogens of LRTI.

In bivariate analysis the variables; age range of 18-35 years (COR $=3.886,95 \% \mathrm{Cl}, 1.712-8.821, \mathrm{p}=.001)$ and $36-49$ years $(\mathrm{COR}=2.773,95 \% \mathrm{Cl}, 1.151-6.677, \mathrm{p}$ $=.023$ ); educational status who were 1-8 grade students (COR=.596, 95\% Cl, .296-1.199, $\mathrm{P}=.147)$, marital status those were married $(\mathrm{COR}=.509$, 95\% $\mathrm{Cl}$, $.305-.850, \mathrm{P}=.010$ ), monthly income with 2001-3000 (COR=.336, 95\% Cl, .070-1.606, $\mathrm{P}=.172$ ) and $>5000$ (COR=.222, 95\%(.038-1.298, $\mathrm{P}=.095)$. In addition those who were non-cigarette smoker (COR=1.984, 95\% Cl, .969-4.062, $\mathrm{P}=.061)$, non-alcohol drinker ( $\mathrm{COR}=1.652,95 \% \mathrm{Cl}, .927-2.945$, $\mathrm{P}=.089)$, had no chronic heart disease $(\mathrm{COR}=1.638,95 \% \mathrm{Cl}, .804-3.334, \mathrm{P}=.174)$ and $\mathrm{HIV}$ result of negative (COR=.749,95\% $\mathrm{Cl}, .486-1.154, \mathrm{P}=.190)$, participants who were unscreened for $\mathrm{HIV}$ at all (COR=.420, $95 \% \mathrm{Cl}, .184-.957, \mathrm{P}=.039)$ were candidate variable for multivariate analysis with $\mathrm{p}$-value $<.250$.

However, in multivariate analysis the age range 18-35 years ( $\mathrm{AOR}=3.856,95 \% \mathrm{Cl}, 1.584-9.387, \mathrm{P}=.003), 36-49$ years $(\mathrm{AOR}=3.136,95 \% \mathrm{Cl}, 1.247-7.888, \mathrm{P}=.015)$, marital status those who are married $(\mathrm{AOR}=.450,95 \% \mathrm{Cl}, .207-.980, \mathrm{p}=.044)$, occupation in government $(\mathrm{AOR}=6.303,95 \% \mathrm{Cl}, 1.508-26.341, \mathrm{P}=.012)$ and monthly income $>5000(\mathrm{AOR}=.060,95 \% \mathrm{Cl}, .007-.526, \mathrm{P}=.011)$ were the only statically significant associated risk factors for LRTI [Table4]. 
Table 4 Associated factors of culture-positive sputum sample of a patient with community acquired LRTI at HUCSH, 2019 (n=406). 


\begin{tabular}{|c|c|c|c|c|c|c|c|}
\hline \multirow[b]{2}{*}{ Variables } & & \multicolumn{2}{|c|}{ Growth } & \multirow[b]{2}{*}{$\operatorname{coR}(95 \% \mathrm{Cl})$} & \multirow[b]{2}{*}{$\mathrm{p}$-value } & \multirow[b]{2}{*}{ AOR(95\%Cl) } & \multirow[b]{2}{*}{ P-value } \\
\hline & & Yes (\%) & No $(\%)$ & & & & \\
\hline \multirow[t]{2}{*}{ Sex } & Male & $87(64.0)$ & $159(58.9)$ & $.807(.527-1.235)$ & 0.323 & & \\
\hline & Female & $49(36.0)$ & $111(41.1)$ & 1 & & & \\
\hline \multirow[t]{5}{*}{ Age(year) } & $18-35$ & $64(47.1)$ & $171(63.3)$ & \multirow{3}{*}{$\begin{array}{l}3.886(1.712- \\
8.821) \\
2.773(1.151- \\
6.677)\end{array}$} & 0.001 & $3.856(1.584-9.387)$ & $0.003^{\star}$ \\
\hline & $36-49$ & $32(23.5)$ & $61(22.6)$ & & 0.023 & $3.136(1.247-7.888)$ & $0.015^{\star}$ \\
\hline & $50-64$ & $24(17.6)$ & $27(10.0)$ & & 0.307 & $1.717(.637-4.631)$ & 0.285 \\
\hline & $>65$ & $16(11.6)$ & $11(4.1)$ & $\begin{array}{l}1.636(.636- \\
4.207)\end{array}$ & & & \\
\hline & & & & 1 & & & \\
\hline \multirow[t]{2}{*}{ Residence } & Rural & $62(45.6)$ & $119(44.1)$ & 1 & & & \\
\hline & Urban & $74(54.4)$ & $151(55.9)$ & $.772(.703-1.609)$ & 0.772 & & \\
\hline \multirow[t]{4}{*}{ Educational status } & \multirow{2}{*}{$\begin{array}{l}\text { No formal } \\
\text { education }\end{array}$} & $48(35.3)$ & $94(34.8)$ & $.703(.348-1.419)$ & 0.326 & $.613(.194-1.931)$ & 0.403 \\
\hline & & $53(39.0)$ & $88(32.6)$ & $.596(.296-1.199)$ & 0.147 & $.427(.146-1.248)$ & 0.120 \\
\hline & \multirow{2}{*}{$\begin{array}{l}\text { 1-8 grade } \\
\text { 9-12 grade } \\
\text { Diploma \& above }\end{array}$} & $21(15.4)$ & $49(18.1)$ & $.838(.378-1.857)$ & 0.663 & $.556(.188-$ & 0.289 \\
\hline & & $14(10.3)$ & $39(14.4)$ & 1 & & & \\
\hline \multirow[t]{2}{*}{ Marital status } & Single & $24(17.6)$ & $80(29.6)$ & 1 & & & \\
\hline & Married & $112(82.4)$ & 190(70.4) & $.509(.305-.850)$ & 0.010 & $.450(.207-.980)$ & $0.044^{*}$ \\
\hline \multirow[t]{6}{*}{ Occupation } & Farmer & $31(22.8)$ & $58(21.5)$ & $.689(.325-1.462$ & 0.332 & $2.661(.841-8.413)$ & 0.096 \\
\hline & Government & $11(8.1)$ & $32(11.9)$ & \multirow{2}{*}{$\begin{array}{l}1.072(.428- \\
2.687)\end{array}$} & 0.882 & $6.303(1.508-26.341)$ & $0.012^{*}$ \\
\hline & Private & $34(25.0)$ & $59(21.9)$ & & 0.239 & $2.425(.882-6.668)$ & 0.086 \\
\hline & Housewife & $41(30.1)$ & $71(26.3)$ & \multirow{2}{*}{$\begin{array}{l}.639(.304-1.345) \\
.638(.309-1.315)\end{array}$} & 0.223 & $2.048(.656-6.392)$ & 0.217 \\
\hline & & 14(10.3) & $38(14.1)$ & & 0.842 & $3.192(.709-14.365)$ & 0.131 \\
\hline & Jobless & $5(3.7)$ & $12(4.4)$ & 1 & & & \\
\hline \multirow[t]{6}{*}{ Monthly income } & $<1000$ & $38(27.9)$ & $88(32.6)$ & $.463(.097-2.215)$ & 0.335 & $.461(.083-2.551)$ & 0.375 \\
\hline & $1000-2000$ & 26(19.1) & $53(19.6)$ & $.408(.083-1.997)$ & 0.268 & $.443(.076-2.578)$ & 0.365 \\
\hline & $2001-3000$ & $44(32.4)$ & $74(27.4)$ & $.336(.070-1.606)$ & 0.172 & $.310(.055-1.752)$ & 0.185 \\
\hline & $3001-5000$ & $17(12.5)$ & $35(13.0)$ & $.412(.081-2.091)$ & 0.285 & $.265(.043-1.61)$ & 0.150 \\
\hline & $>5000$ & $9(6.6)$ & $10(3.7)$ & $.222(.038-1.298)$ & 0.095 & $.060(.007-.526)$ & 0.011 \\
\hline & $\begin{array}{l}\text { No monthly } \\
\text { income }\end{array}$ & $2(1.5)$ & $10(3.7)$ & 1 & & & \\
\hline \multirow[t]{2}{*}{ Cigarette Smoking } & Smoker & 16(11.8) & $17(6.3)$ & 1 & & & \\
\hline & No smoker & $120(88.4)$ & 253(93.7) & $\begin{array}{l}1.984(.969- \\
4.062)\end{array}$ & 0.061 & $2.274(.810-6.385)$ & 0.119 \\
\hline \multirow[t]{2}{*}{ Alcohol drinking } & Yes & $24(17.6)$ & $31(11.5)$ & 1 & & & \\
\hline & No & $112(82.4)$ & $239(88.5)$ & $\begin{array}{l}1.652(.927- \\
2.945)\end{array}$ & 0.089 & $1.112(.468-2.642)$ & 0.809 \\
\hline \multirow{2}{*}{$\begin{array}{l}\text { Crowded living } \\
\text { condition }\end{array}$} & Yes & 16(11.8) & $36(13.3)$ & 1 & & & \\
\hline & No & $120(88.2)$ & 234(86.7) & $\begin{array}{l}1.154(.615- \\
2.164)\end{array}$ & 0.655 & & \\
\hline \multirow{2}{*}{$\begin{array}{l}\text { History of previous } \\
\text { TB }\end{array}$} & Yes & $9(6.6)$ & $17(6.3)$ & 1 & & & \\
\hline & No & 127(93.4) & 253(93.7) & $\begin{array}{l}1.055(.457- \\
2.432)\end{array}$ & 0.901 & & \\
\hline \multirow[t]{2}{*}{ Chronic heart disease } & Yes & $15(11.0)$ & 19(7.0) & 1 & & & \\
\hline & No & $121(89.0)$ & $251(93.0)$ & $\begin{array}{l}1.638(.804- \\
3.334)\end{array}$ & 0.174 & $1.530(.688-3.401)$ & 0.297 \\
\hline $\begin{array}{l}\text { Previous HIV } \\
\text { screening }\end{array}$ & Positive & 13(9.6) & $13(4.8)$ & 1 & & & \\
\hline
\end{tabular}

Page 7/12 
$\mathrm{Cl}=$ Confidence Interval, OR=Odds Ratio

\section{Antimicrobial susceptibility patterns of bacterial isolates}

\section{Gram positive bacteria}

In this study, Gram-positive were sensitive to clindamycin $26(76.5 \%)$ and erythromycin $18(55.9 \%)$ however, they showed resistant to tetracycline $20(58.8 \%)$. S. aureus was sensitive to cefoxitin 19(90.6\%), gentamycin 16(76.2\%) and ciprofloxacin 15(71.4\%). S. aureus was resistant to tetracycline $13(61.9 \%)$ and cotrimoxazole 10(47.6\%). S. pneumoniae were sensitive to clindamycin 8(88.9\%), penicillin 7(77.8\%), erythromycin 6(66.7\%), but S. pneumoniae was resistant to tetracycline 6(66.7\%). All S. pyogenes isolates were 3(100\%) sensitive to tetracycline, ampicillin, clindamycin, erythromycin and penicillin [Table5].

\section{Gram negative bacteria}

Gram-negative bacteria were sensitive to cefepime 93(86.0\%), ciprofloxacin 84(77.8\%), ampicillin 13(20.3\%), augmentin 18(28.1\%) and ceftazidime 49(45.4\%). K. pneumoniae was sensitive to ciprofloxacin 33(91.7\%), cefepime $30(83.3 \%)$ and cefoxitin $30(83.3 \%)$ and $K$. pneumoniae was resistant

to augmentin 20(56.6\%), ceftazidime 19(52.8\%) and ampicillin 19(52.8\%) . Pseudomonas spp was sensitive to cefepime 23(92.0\%), ciprofloxacin 17(68.0\%), gentamycin 17(68.0\%), imipenem 16(64.0\%) while Pseudomonas spp was resistant to ceftazidime 17(68.0\%). E. coli were susceptible to cefoxitin 18(81.1\%), cefepime $17(77.3 \%)$ and show resistant to ampicillin 19(86.4\%). Acinetobacter spp was sensitive to cefepime 17(89.5\%), ceftriaxone 16(84.2\%), gentamycin $16(84.2 \%)$ but resistant to cotrimoxazole 10(52.6\%). All Enterobacter spp and Citrobacter spp were 100\% sensitive to cefepime, tetracycline, ceftriaxone and ciprofloxacin antibiotics [Table6].

\section{Multiple drug resistance (MDR) patterns of the isolates}

In this study, the overall multidrug resistance (MDR) bacteria were 47/142(33.1\%). E. coli was the most isolate that showed MDR $13(59.1 \%)$ followed by $S$. aureus 12(57.1\%) and K. pneumoniae 12(33.3\%). Majority of bacterial isolates were resistant to three classes of antibiotics 25(17.6\%)[Table7].

Table 5 Antibiotic susceptibility patterns of gram-positive bacterial isolates from lower respiratory tract infection at HUCSH, 2019

\begin{tabular}{|c|c|c|c|c|c|c|c|c|c|c|c|c|}
\hline & & Antibiotic & (\%) & & & & & & & & & \\
\hline Isolates & Pattern & CXT & TAT & AMP & CPR & COT & CLD & ERY & $\mathrm{GN}$ & PEN & CFP & CAF \\
\hline \multirow[t]{3}{*}{ S. aureus $(\mathrm{N}=21)$} & $S$ & 19(90.6) & $3(14.3)$ & & 15(71.4) & $9(42.9)$ & $14(66.7)$ & $10(47.6)$ & $16(76.2)$ & $9(45.0)$ & & \\
\hline & I & $1(4.7)$ & $5(23.8)$ & NR & $1(4.8)$ & $2(9.5)$ & 0 & 0 & $3(14.3)$ & $2(10.0)$ & NR & NR \\
\hline & $\mathrm{R}$ & $1(4.7)$ & 13(61.9) & & $5(23.8)$ & $10(47.6)$ & 7(33.3) & $11(52.4)$ & $2(9.5)$ & $9(45.0)$ & & \\
\hline \multirow{3}{*}{$\begin{array}{l}\text { S. pneumoniae } \\
(\mathrm{N}=9)\end{array}$} & $S$ & & $2(22.2)$ & & & & $8(88.9)$ & $6(66.7)$ & NR & $7(77.8)$ & & \\
\hline & I & NR & $1(11.1)$ & NR & NR & NR & 0 & $2(22.2)$ & & 0 & NR & NR \\
\hline & $\mathrm{R}$ & & $6(66.7)$ & & & & $1(11.1)$ & $1(11.1)$ & & $2(22.2)$ & & \\
\hline \multirow[t]{3}{*}{ S. pyogene $(\mathrm{N}=3)$} & $S$ & & $3(100)$ & $3(100)$ & & & $3(100)$ & $3(100)$ & NR & $3(100)$ & $2(66.7)$ & \\
\hline & I & NR & 0 & 0 & NR & NR & 0 & 0 & & 0 & 0 & NR \\
\hline & $\mathrm{R}$ & & 0 & 0 & & & 0 & 0 & & 0 & $1(33.3)$ & \\
\hline \multirow{3}{*}{$\begin{array}{l}\text { Enterococcus } \\
\operatorname{spp}(\mathrm{N}=1)\end{array}$} & $S$ & NR & 0 & NR & $1(100)$ & 0 & $1(100)$ & 0 & & & & 0 \\
\hline & I & & 0 & & 0 & 0 & 0 & 0 & NR & NR & NR & 0 \\
\hline & $\mathrm{R}$ & & $1(100)$ & & 0 & $1(100)$ & 0 & $1(100)$ & & & & $1(100)$ \\
\hline \multirow[t]{3}{*}{ Total (34) } & $S$ & 19(90.6) & $8(23.5)$ & $3(100)$ & $16(72.0)$ & $9(40.0)$ & $26(76.5)$ & 19(55.9) & $16(76.2)$ & $19(57.6)$ & $3(100)$ & 0 \\
\hline & I & $1(4.7)$ & $6(17.6)$ & 0 & $1(4.5)$ & $2(10.0)$ & 0 & $1(2.9)$ & $3(14.3)$ & $2(6.0)$ & 0 & 0 \\
\hline & $\mathrm{R}$ & $1(4.7)$ & $20(58.8)$ & 0 & $5(22.7)$ & $11(50.0)$ & $8(23.5)$ & $14(41.2)$ & $2(9.5)$ & 11(33.3) & 0 & $1(100)$ \\
\hline
\end{tabular}

NR-not recommended, CXT-cefoxitin, AMP-ampicillin, CEF-cefepime, TAT-tetracycline, CPR-ciprofloxacin, COT-cotrimoxazole- erythromycin, PEN-penicillin, CAFchloramphenicol, CLD-clindamycin, GN-gentamycin, S- sensitivity, l-intermediate, R-resistance

Table 6 Antibiotic susceptibility pattern of gram-negative bacterial isolates of an adult patient with lower respiratory tract infection at HUCSH, 2019 


\begin{tabular}{|c|c|c|c|c|c|c|c|c|c|c|c|c|}
\hline \multirow[t]{2}{*}{ Isolates } & \multicolumn{12}{|c|}{ Antibiotics (\%) } \\
\hline & Pattern & CFP & CAZ & CXT & TAT & CTR & AMP & AUG & CPR & COT & GN & IMP \\
\hline \multirow{3}{*}{$\begin{array}{l}\text { K. } \\
\text { pneumoniae } \\
(\mathrm{N}=36)\end{array}$} & $S$ & $30(83.3)$ & 13(36.1) & $30(83.3)$ & 15(41.7) & $24(66.7)$ & $9(25.0)$ & 12(33.3) & 33(91.7) & 19(52.8) & & \\
\hline & I & 0 & $4(11.1)$ & 0 & $4(11.1)$ & $4(11.1)$ & $8(22.2)$ & $4(11.1)$ & 0 & $1(2.8)$ & NA & NR \\
\hline & $\mathrm{R}$ & $6(16.7)$ & $19(52.8)$ & $6(16.7)$ & $17(47.2)$ & $8(22.2)$ & 19(52.8) & $20(56.6)$ & $3(8.3)$ & $16(44.4)$ & & \\
\hline \multirow{3}{*}{$\begin{array}{l}\text { Pseudomonas } \\
\operatorname{spp}(\mathrm{N}=25)\end{array}$} & $S$ & 23(92.0) & $6(24.0)$ & & & & & & $17(68.0)$ & & $17(68.0)$ & $16(64.0)$ \\
\hline & I & 0 & $2(8.0)$ & NR & NR & NR & NR & NR & 0 & NR & $4(16.0)$ & $2(8.0)$ \\
\hline & $\mathrm{R}$ & $2(8.0)$ & $17(68.0)$ & & & & & & $8(32.0)$ & & $4(16.0)$ & $7(28.0)$ \\
\hline \multirow[t]{3}{*}{ E.coli $(\mathrm{N}=22)$} & $S$ & 17(77.3) & 11(50.0) & 18(81.1) & $3(13.6)$ & $14(63.6)$ & $3(13.6)$ & $5(22.7)$ & 13(59.1) & $10(40.9)$ & & \\
\hline & I & $1(4.5)$ & $2(9.1)$ & $2(9.1)$ & $5(22.7)$ & 0 & 0 & $1(4.5)$ & 0 & $1(45.5)$ & NA & NR \\
\hline & $\mathrm{R}$ & $4(18.2)$ & $9(40.9)$ & $2(9.1)$ & 14(63.6) & $8(36.4)$ & 19(86.4) & $16(72.7)$ & $9(40.9)$ & $11(4.5)$ & & \\
\hline \multirow{3}{*}{$\begin{array}{l}\text { Acinetobacter } \\
(\mathrm{N}=19)\end{array}$} & $S$ & 17(89.5) & $13(68.4)$ & & & $16(84.2)$ & & & 15(78.9) & 6(31.6) & $16(84.2)$ & 12(63.2) \\
\hline & I & 0 & $1(5.3)$ & NR & NR & $2(10.5)$ & NR & NR & $1(5.3)$ & $3(15.8)$ & $2(10.5)$ & $2(10.5)$ \\
\hline & $\mathrm{R}$ & $2(10.5)$ & $5(26.3)$ & & & $1(5.3)$ & & & $3(15.3)$ & $10(52.6)$ & $1(5.3)$ & $5(26.3)$ \\
\hline \multirow{3}{*}{$\begin{array}{l}\text { Enterobacter } \\
\operatorname{spp}(\mathrm{N}=4)\end{array}$} & $S$ & $4(100.0)$ & $4(100)$ & $3(75.0)$ & $4(100)$ & $4(100)$ & $1(25)$ & $1(25)$ & $4(100)$ & $2(50)$ & & \\
\hline & I & 0 & 0 & 0 & 0 & 0 & 0 & 0 & 0 & 0 & NA & NR \\
\hline & $\mathrm{R}$ & 0 & 0 & $1(25.0)$ & 0 & 0 & $3(75)$ & $3(75)$ & 0 & $2(50)$ & & \\
\hline \multirow{3}{*}{$\begin{array}{l}\text { Citrobacter } \\
\text { spp }(\mathrm{N}=2)\end{array}$} & $S$ & $2(100.0)$ & 0 & 1(50.0) & $2(100.0)$ & $2(100.0)$ & 0 & 0 & $2(100.0)$ & $1(50)$ & & \\
\hline & 1 & 0 & 0 & 0 & 0 & 0 & 0 & 0 & 0 & 0 & NA & NR \\
\hline & $\mathrm{R}$ & 0 & $2(100.0)$ & $1(50.0)$ & 0 & 0 & $2(100.0$ & $2(100.0)$ & 0 & $1(50)$ & & \\
\hline \multirow{3}{*}{$\begin{array}{l}\text { Total } \\
(\mathrm{N}=108)\end{array}$} & $S$ & $93(86.0)$ & $49(45.4)$ & $52(81.3)$ & 23(35.9) & $60(72.3)$ & 13(20.3) & 18(28.1) & $84(77.8)$ & $38(45.8)$ & $33(75.0)$ & $28(63.6)$ \\
\hline & I & $1(0.9)$ & $9(8.3)$ & $2(3.1)$ & 10(15.6) & $6(7.2)$ & $8(12.5)$ & $5(7.8)$ & $1(0.9)$ & $5(6.0)$ & $6(13.6)$ & $4(9.1)$ \\
\hline & $\mathrm{R}$ & 14(13.0) & $50(46.3)$ & $9(15.6)$ & $31(48.4)$ & $17(20.5)$ & $43(67.2)$ & $41(64.0)$ & 23(21.3) & $40(48.2)$ & $5(11.4)$ & $12(27.3)$ \\
\hline
\end{tabular}

NA-not applied, NR-not recommended, AUG-augmentin, CAZ- ceftazidime, CXT-cefoxitin, AMP- ampicillin, CEF-cefepime, TAT-tetracycline, CTR-ceftriaxone, CPRciprofloxacin, GN- gentamycin, IMP- imipenem, S- sensitivity, I- intermediate, R- resistance

Table 7 multi-drug resistant rates of bacterial isolates among adult patient with lower respiratory tract infection at HUCSH, 2019

\begin{tabular}{|lllllllll|}
\hline Isolate & $\mathrm{R}(\%)$ & $\mathrm{R} 1(\%)$ & $\mathrm{R} 2(\%)$ & $\mathrm{R} 3(\%)$ & $\mathrm{R} 4(\%)$ & $\mathrm{R} 5(\%)$ & $\geq \mathrm{R} 6(\%)$ & Total $(\%)$ \\
\hline K. pneumoniae $(\mathrm{N}=36)$ & $3(8.3)$ & $7(19.4)$ & $13(36.1)$ & $7(19.4)$ & $3(8.3)$ & $2(5.6)$ & 0 & $12(33.3)$ \\
\hline S. aureus $(\mathrm{N}=21)$ & $3(14)$ & $2(9.5)$ & $4(19.0)$ & $6(28.6)$ & $3(14.3)$ & $2(9.5)$ & $1(4.7)$ & $12(57.1)$ \\
\hline E. coli $(\mathrm{N}=22)$ & $3(13.6)$ & $3(13.6)$ & $4(18.2)$ & $3(13.6)$ & $3(16.6)$ & 0 & $6(27.3)$ & $12(54.5)$ \\
\hline Pseudomonas $\operatorname{spp}(\mathrm{N}=25)$ & $4(16.0)$ & $7(28.0)$ & $10(40.0)$ & $4(16.0)$ & 0 & 0 & 0 & $4(16.0)$ \\
\hline Acinetobacter $\operatorname{spp}(\mathrm{N}=19)$ & $6(31.6)$ & $5(26.3)$ & $6(31.6)$ & $2(10.5)$ & 0 & 0 & 0 & $2(10.5)$ \\
\hline S. pneumoniae $(\mathrm{N}=9)$ & $2(22.2)$ & $5(55.5)$ & $1(11.1)$ & $1(11.1)$ & 0 & 0 & $1(11.1)$ \\
\hline Enterobacter $\operatorname{spp}(\mathrm{N}=4)$ & 0 & $1(25.0)$ & $2(50.0)$ & $1(25.0)$ & 0 & 0 & 0 & $1(25.0)$ \\
\hline S. pyogene $(\mathrm{N}=3)$ & $3(100)$ & 0 & 0 & 0 & 0 & 0 & 0 & $1(50.0)$ \\
\hline Citrobacter $\operatorname{spp}(\mathrm{N}=2)$ & 0 & 0 & $1(50.0)$ & $1(50.0)$ & 0 & 0 & 0 & $1(100)$ \\
\hline Enterococcus $\operatorname{spp}(\mathrm{N}=1)$ & 0 & 0 & 0 & 0 & $1(100)$ & 0 & 0 & $46 / 142(32.4)$ \\
\hline Total $(\mathrm{N}=142)$ & $24(17.0)$ & $30(21.1)$ & $41(28.9)$ & $25(17.6)$ & $10(7.0)$ & $4(2.8)$ & $7(4.9)$ & 4 \\
\hline
\end{tabular}

Key: R0- no antibiotic resistance, R1-resistance to one, R2-resistance to two, R3-resistance to three, R4-resistance to four, R5-resistance to five, and ZR6resistance to six and more than six antibiotics families. 


\section{Discussion}

In this study, the overall of sputum culture positive of community acquired LRTI among adult patients was 136(33.5\%). This finding is comparable to result reported from Arba Minch, Ethiopia [6] 40\% but lower than Felege Hiwot [13] 40.3\%, Jimma, Ethiopia, [14] 45\%, Zagazig, Egypt, 50.4\% [15] and South India, 55.3\% [16]. However, it was higher than studies result conducted in Central Kerala, India [17], Sri Lanka, Colombo, [1] and Central Nepal, [18] which were $26.34 \%, 29.4 \%$ and $30.4 \%$ respectively. This difference may be due to geographical location, study period and socioeconomic status of the study population. The distribution of respiratory infections varies between populations and countries, depending on geographical, climatic and socioeconomic conditions [3].

K. pneumoniae $25.4 \%$ was the most frequent bacterial isolate followed by Pseudomonas spp $17.6 \%$, E. coli $15.5 \%$ and $S$. aureus $14.8 \%$. This finding is similar to the study conducted in Assuit, Egypt [19], in which K. pneumoniae 58\% was the most frequent isolate followed by $P$. aeruginosa $28 \%$. This finding also agrees with Janakpur, Nepal 27.0\% [20], Zagazig, Egypt, 10.4\% [15] and 31.5\% South India, [16] in which K. pneumoniae was the commonest detected of LRTI. In contrast, in the previous studies in Ethiopian such as Felege Hiwot 35.9\% [13] Jimma 12.8\% [14] and Arba Minch 11.8\% [6] S. pneumoniae was the predominant bacterial pathogen of culture-positive sputum.

S. aureus which accounts $14.8 \%$ is higher than $8.8 \%$ Arba Minch [6] and 10.5\% Jimma [14], but all most equal to Felege Hiwot [13] 14.4\% in Ethiopian studies. When compared other parts of world studies, it is higher than Central India 3.55\% [21], Zigazig, Egypt 4.7\% [15] and Waziristan, Pakistan 5.9\% [22]. However lower than the study conducted in Janakpur, Nepal 20.8\% [20].

In our study, K. pneumoniae shows a high rate of sensitive to ciprofloxacin $91.7 \%$, cefepime and cefoxitin $83.3 \%$ each and resistant to augmentin $56.6 \%$, ceftazidime and ampicillin $52.8 \%$ each and tetracycline $47.2 \%$. In contrast to this finding two other studies in Ethiopia showed, $K$. pneumoniae exhibit high rates of resistance to tetracycline $100 \%$ and augmentin $96.7 \%$ [13] and Regasa et al reported as K. pneumoniae was $100 \%$ resistant to ampicillin and tetracycline [14].

The current study revealed that $S$. aureus was highly sensitive to cefoxitin $90.6 \%$, gentamycin $76.2 \%$ and ciprofloxacin $71.4 \%$. However, $S$. aureus was resistant to tetracycline $61.9 \%$, cotrimoxazole $47.6 \%$ and penicillin $45 \%$. In agreement to this, a study from Jimma, Ethiopia [14] reported $S$. aureus as high resistance to tetracycline $100 \%$, penicillin and cotrimoxazole $81.3 \%$ to each, erythromycin $75 \%$. Low resistance was observed for ciprofloxacin $31.3 \%$ and gentamycin $31.5 \%$. In contrast to this high rates of sensitivity reported from Karachi, Pakistan to gentamicin $66.7 \%$ [23].

The overall magnitude of multidrug resistance(MDR) was 33.1\% which is lower than other Ethiopian studies Jimma [14], Arba Minch [24] and Felege Hiwot [13] $60.3 \%, 62.7 \%$ and $76 \%$ respectively. However, it is higher than Cairo, Egypt [25] 26.4\%. This variation is may be due to the difference in the type of tested drugs and bacterial isolated. E. coli was the most isolate that showed MDR $59.1 \%$ followed by $S$. aureus $57.1 \%$ and $K$. pneumoniae $33.3 \%$ in this study. In a similar study in Cairo, Egypt [25] those organisms also exhibit MDR, K. pneumoniae $37.1 \%$, E. coli $24.2 \%$, S. aureus $14.5 \%$.

Our study assessed the predisposing factors for culture-positive sputum sample among adult patient with LRTI. Accordingly, as compared to age > 65 years those with age group 18-35 years 3.856 times and age group 36-49 years 3.136 times at risk of culture-positive for LRTI. In contrast to our finding, most studies reported that elder age is a significant risk factor for LRTI, with the risk growing especially for people $>65$ age [26, 27]. This difference is may be due to the 18-49 is working age and may have exposure to risk factor such as cigarette smoking, alcohol drinking and engaging in overcrowded area. In fact in developing countries, the main burden of hospitalized patients with CAP is among adults in the working-age but in developed countries in older patients with co-morbidity [28]. In this case, Ethiopia is one of the developing countries. Similarly, a study conducted on, risk factors for community-acquired pneumonia in adults: recommendations for its prevention, in Spain, state that age is a possible nonlinear effect, with older age as a risk factor for CAP [29].

Moreover, as compared to being single, those who are married $45 \%$ decreased for being culture-positive sputum of adult patient of LRTI. This may due to the single marital status individual at adult age have the exposure to alcohol drinking, cigarette smoking and chronic disease such as HIV. In fact, alcoholismrelated conditions and comorbidities increased among single individual[30]. Similarly, the study from Spain [29], suggested that being married or in a partnership is a protective factor for CAP in comparison to being single, widowed or separated.

\section{Conclusion}

K. pneumoniae was the predominant of lower respiratory tract infection pathogen followed by Pseudomonas species. Cefepime, ciprofloxacin and cefoxitin were the most effective antibiotics against gram-negative bacteria while clindamycin for gram-positive. However, the isolates showed resistance to common antibiotics ampicillin, augmentin, ceftazidime and tetracycline. Working age group and single marital status are associated with an increased risk of culture positive sputum of adult patient of lower respiratory tract infection. Therefore, culture and susceptibility test is vital for appropriate management of lower respiratory tract infection in the study area.

\section{Abbreviations}

AFB: Acid Fast Bacilli; ATCC: American Type Culture Collection; CAP: Community Acquired Pneumonia; CLSI: Clinical and Laboratory Standards Institute; HUCSH: Hawassa University Comprehensive Specialized Hospital; IRB: Institution Research Board; LRTIs: Lower Respiratory Tract Infections; MDR: Multi Drug Resistance; OPD: Out Patient Department; SPSS: Statistical Package for Social Science

\section{Declarations}

\section{Ethics approval and consent to participate}


Ethical approval was obtained from the institutional review board of Hawassa University, College of medicine and health sciences (Ref No: IRB/231/11). Informed written consent was obtained from all participants. All methods were carried out in accordance with relevant guidelines and regulations. Clinicians were communicated the findings of culture and sensitivity tests.

\section{Consent for publication}

Individual data such as images and videos did not accompany this particular manuscript and hence consent for publication is not applicable.

\section{Availability of data and materials}

The datasets used and analyzed during the current study are available from the corresponding author on reasonable request.

\section{Competing interests}

The authors declare that they have no competing interests for this work.

\section{Funding}

Not applicable.

\section{Authors' contributions}

M.D.O., T.A.B. and A.B.G conceived the study and participated in data analysis. T.A.B. and A.B.G carried out the lab work, M.D.O. wrote the manuscript. All authors read and approved the final manuscript.

\section{Acknowledgments}

We are grateful to Hawassa University Comprehensive Specialized Hospital microbiology laboratory staffs and study participants for their contribution to this research work.

\section{References}

1. Amarasinghe N, Athavan M, Jayamanne D, Rajapakshe Y, Sadikeen A, Gunasekara K, Fernando A, Karunanayake L. Bacterial profile and antibiotic susceptibility pattern of adult lower respiratory tract infections in Colombo, Sri Lanka. Journal of Health Social Sciences. 2018;3(1):27-36.

2. Bigna TS, Nzouankeu JJ, Fonkoua A, Nansseu M-C, Ndangang JR, Kenmoe MS, Penlap S, Njouom VB. Prevalence of respiratory bacterial infections in people with lower respiratory tract infections in Africa: the BARIAFRICA systematic review and meta-analysis protocol. BMJ open. 2018;8(9):e023592.

3. Tchatchouang S, Nzouankeu A, Kenmoe S, Ngando L, Penlap V, Fonkoua M-C, Pefura-Yone E-W, Njouom R: Bacterial Aetiologies of Lower Respiratory Tract Infections among Adults in Yaoundé, Cameroon. BioMed research international 2019, 2019.

4. Aston SJ. Pneumonia in the developing world: C haracteristic features and approach to management. Respirology. 2017;22(7):1276-87.

5. Vishwanath S, Chawla K, Gopinathan A. Multidrug resistant Gram-negative bacilli in lower respiratory tract infections. Iran J Microbial. $2013 ; 5(4): 323-7$.

6. Regasa B. Aetiology of bacterial pathogens from adult patients with community-acquired pneumonia in Arba Minch Hospital, South Ethiopia. Science Journal of Clinical Medicine. 2014;3(3):33-6.

7. Imaz M, Allassia S, Aranibar M, Gunia A, Poggi S, Togneri A, Wolff L. Group of Implementation of Fluorescence Microscopy: Performance of LED fluorescence microscopy for the detection of acid-fast bacilli from respiratory samples in peripheral laboratories in Argentina. Biomédica. 2017;37(2):164-74.

8. Krishna S, Jabeen H, Jeer M: Correlation of Sputum Gram Stain and Sputum Culture for Respiratory Tract Infections in a Tertiary Care Hospital, Ballari, India. IntJCurrMicrobiolAppSci 2017, 6(6):3008-3012.

9. Cheesbrough M: District laboratory practice in tropical countries: Cambridge university press; 2006.

10. Keerthana S, Appalaraju MT. Haemophilus influenzae in Community Acquired Infections. International Journal of Current Microbiology Applied Sciences. 2017;6(4):926-37.

11. CLSI. Performance standards for antimicrobial susceptibility testing CLSI supplement M100S Wayne. In., vol. 39, 29th ed. edn; 2019.

12. Bursac Z, Gauss CH, Williams DK, Hosmer DW. Purposeful selection of variables in logistic regression. Source Code Biol Med. 2008;3:17-7.

13. Temesgen D, Bereded F, Derbie A, Biadglegne F. Bacteriology of community acquired pneumonia in adult patients at Felege Hiwot Referral Hospital, Northwest Ethiopia: a cross-sectional study. Antimicrobial Resistance Infection Control. 2019;8(1):101.

14. Regasa B, Yilma D, Sewunet T, Beyene G. Antimicrobial susceptibility pattern of bacterial isolates from community-acquired pneumonia patients in Jimma University specialized hospital, Jimma, Ethiopia. Saudi Journal for Health Sciences. 2015;4(1):59.

15. El-Sokkary RH, Ramadan RA, El-Shabrawy M, El-Korashi LA, Elhawary A, Embarak S, Tash RME, Elantouny NG. community acquired pneumonia among adult patients at an egyptian university hospital: bacterial etiology, susceptibility profile and evaluation of the response to initial empiric antibiotic therapy. Infection drug resistance. 2018;11:2141-50.

16. Ramana K, Kalaskar A, Rao M, Rao SD. Aetiology and antimicrobial susceptibility patterns of lower respiratory tract infections (LRTI's) in a rural tertiary care teaching hospital in Karimnagar, South India. Am J Infect Dis Microbiol. 2013;1(1):101-5. 
17. Regha I, Sulekha B. Bacteriological profile and antibiotic susceptibility patterns of lower respiratory tract infections in a tertiary care hospital, Central Kerala. International Journal of Medical Microbiology Tropical Diseases. 2018;4(4):186-90.

18. Shrestha S, Acharya A. Lower respiratory tract pathogens and their antimicrobial susceptibility pattern in a medical hospital of central Nepal. International Journal of Biomedical Advance Research. 2013;4(5):335-40.

19. Ghanem MK, Makhlouf HA, Hasan AA, Rashed HG, Khalifa HS. Bacteriological profile of critically ill patients with chronic obstructive pulmonary disease in respiratory intensive care unit in Assuit University Hospital. Egyptian Journal of Bronchology. 2019;13(3):343.

20. Khushbu Y, Satyam P: Bacteriological Profile of Lower Respiratory Tract Infection (LRTI) among HIV Seropositive Cases in Central Terai of Nepal. IntJCurrMicrobiolAppSci 2015, 4(11):431-442.

21. Bajpai T, Shrivastava G, Bhatambare G, Deshmukh A, Chitnis V. Microbiological profile of lower respiratory tract infections in neurological intensive care unit of a tertiary care center from Central India. Journal of basic clinical pharmacy. 2013;4(3):51.

22. Shah SN, Basit BUA, Begum A, Tabassum A, Zafar S, Saleha S. Prevalence and susceptibility patterns of bacteria causing respiratory tract infections in North Waziristan, Pakistan. Pak J Pharm Sci. 2016;29(2):701-6.

23. Zafar A, Hussain Z, Lomama E, Sibille S, Irfan S, Khan E. Antibiotic susceptibility of pathogens isolated from patients with community-acquired respiratory tract infections in pakistan the active study. J Ayub Med Coll Abbottabad. 2008;20(1):1-9.

24. Regasa B. Drug resistance patterns of bacterial pathogens from adult patients with pneumonia in Arba Minch Hospital, South Ethiopia. Global Journals Inc (USA). 2014;14(5):2249-4618.

25. Aboshanab K, Abdelaziz S, Hassouna NA, Aboulwafa MM: Antimicrobial resistance pattem of some bacterial pathogens involved in lower respiratory tract infections in Egypt. Acta Microbiologica, 6 (1: 1) 2015.

26. Moradi LM, Eibcheraoui C, Charara R, Khalil I, Afshin A, Kassebaum JN, Collison M, Daoud F, Chew A, Krohn K, et al. Burden of lower respiratory infections in the Eastern Mediterranean Region between 1990 and 2015: findings from the Global Burden of Disease 2015 study. International Journal of Public Health. 2018;63(1):97-108.

27. CDC. CDC Pneumonia (Ventilator-associated [VAP] and non-ventilator-associated Pneumonia [PNEU]) Event. 2019:1-16.

28. Tejada S, Romero A, Rello J. Community-Acquired Pneumonia in Adults: What's New Focusing on Epidemiology, Microorganisms and Diagnosis? Erciyes Medical Journal/Erciyes Tip Dergisi 2018, 40(4).

29. Almirall J, Serra-Prat M, Bolibar I. Risk factors for community-acquired pneumonia in adults: Recommendations for its prevention. Community Acquired Infection. 2015;2(2):32.

30. Mor A, Ulrichsen SP, Svensson E, Berencsi K, Thomsen RW. Does marriage protect against hospitalization with pneumonia? A population-based casecontrol study. Clinical epidemiology. 2013;5:397.

\section{Supplementary Files}

This is a list of supplementary files associated with this preprint. Click to download.

- STROBEchecklistcrosssectionalwordfilled.doc 